Sādhanā Vol. 40, Part 5, August 2015, pp. 1473-1479. (c) Indian Academy of Sciences

\title{
Electrical equivalent model of intermediate band solar cell using PSpice
}

\author{
B LAKEHAL ${ }^{1,2, *}, \mathrm{ZDIBI}^{2}, \mathrm{~N} \mathrm{LAKHDAR}^{2,3}$ and A DENDOUGA ${ }^{4}$ \\ ${ }^{1}$ Department of Electronics, University of Kasdi Merbah Ouargla, \\ Ouargla 30000, Algeria \\ ${ }^{2}$ Advanced Electronics Laboratory, University of Batna, Batna 05000, Algeria \\ ${ }^{3}$ Department of Electronics, University of HL El Oued, El oued 39000, Algeria \\ ${ }^{4}$ Centre de développement des technologies avancées CDTA, Baba Hassen 16303, \\ Algeria \\ e-mail: Lakehal.brahim@univ-ouargla.dz
}

MS received 6 March 2014; revised 20 January 2015; accepted 12 April 2015

\begin{abstract}
Intermediate band solar cells (IBSCS) is a relatively new solar cell concept. The theoretical conversion efficiency limit for IBSCS is predicted to be $63.2 \%$ with blackbody illumination and $65.1 \%$ with AM1.5 spectrum, comparable to the theoretical efficiency for optimized triple junction solar cells with efficiencies of $63.8 \%$ and $67.0 \%$ under blackbody and AM1.5 illumination, respectively. This paper presents a structure of IBSC based on ZnTe:O. The proposed model uses irradiance and temperature as input parameters and the output characteristics of the structure are used as a prototypical example. So, in this work the artificial neural networks (ANNs) and the analog behavior modeling (ABM) of PSpice are implemented in order to model the IBSC. In addition, we have used the bias matrix and the weights matrix obtained by training to establish the IBSCS model on PSPICE simulator. This method represents an advantage by using a small representative database. Therefore, the obtained results of our model can be used in an electrical simulator. Thus, this method can be extended to other solar cells for motivating experimental efforts to realize these promising photovoltaic devices for low cost and high efficiency.
\end{abstract}

Keywords. PSpice simulator; IBSCS; neuronal network; analog behavior modeling $(\mathrm{ABM})$.

\section{Introduction}

Worldwide photo-voltaic (PV) research is continuing in order to produce a low-cost and highefficiency device for solar energy conversion. Power conversion efficiency has been improved for tandem and multi-junction solar cells, but these devices are more complex and are accompanied by higher manufacturing costs. Many techniques have been proposed to increase the efficiency of solar cells. They are based on different processes and properties such as photon recycling,

*For correspondence 
impact ionization, angle dependent selectivity, down-conversion and up-conversion (Schokley \& Queisser 1961; Luque \& Mart1 1997; Bremner \& Honsberg 2012; Luque et al 2013). In recent years, intermediate-band solar cells (IBSCS) have been proposed to exceed efficiency limitations of conventional single-junction cells (Nozik 2006). In these devices, electron states are introduced in the forbidden band gap of a conventional semiconductor to provide three optical absorption bands to respond to incident solar energy. The theoretical conversion efficiency limit for IBSCS is predicted to be $63.2 \%$ with blackbody illumination and $65.1 \%$ with AM1.5 spectrum, comparable to the theoretical efficiency for optimized triple junction solar cells with efficiencies of $63.8 \%$ and $67.0 \%$ under blackbody and AM1.5 illumination, respectively (Luque et al 2001, 2004; Marti \& Luque 2004), Several approaches have been proposed to practically realize an intermediate band solar cell including quantum dots, dopant impurities (often termed impurity-band photovoltaic), and dilute semiconductor alloys (Huan-Liang et al 2008; Khelifia et al 2008; Stanko et al 2008; Lin et al 2009).

Recently, artificial neural networks (ANNs) have been successfully employed in solving complex problems in various fields of applications including pattern recognition, identification, classification, speech, vision, prediction and control systems (Moufdi et al 2012). It is emerged as a highly effective learning technique suitable for performing nonlinear, complex, and dynamic tasks with a high degree of accuracy. Neural models are, therefore, much faster than physics/electro-mechanical models and have a higher accuracy than analytical and empirical models. Furthermore, they are easy to develop for a new device or technology (Kalogirou 2000; Tobías et al 2011; Mekki et al 2007; Lopes \& Lienhardt 2003). The MATLAB interface was used during the design phase and optimization; the results (optimal architecture, bias, and weights of the network) are used for the implementation of the model as a component in the PSpice simulator library. The proposed technique keeps its validity also when applied to other systems for motivating experimental efforts to realize these promising photovoltaic devices.

\section{Intermediate band solar cell modeling}

\subsection{Implementation of IBSCS model}

The schematic structure of ZnTe-based IBSCS is presented in figure 1. Material parameters for $\mathrm{ZnTe}$ are chosen in this analysis as a prototypical material for IBSC due to recent interest in oxygen-doped ZnTe as an active region for IBSC devices.

Neural network is mostly used to find the appropriate solution for the non-linear and complex systems. Among its classes, there is the Multi-Layer perceptron (MLP) which is the most used, where the simpler architecture of MLP contains three layers as shown in figure 2. The input layer receives the external data. The hidden layers contain numerous hidden neurons that receive data from the input layer and send them to the third layer. Finally, the output layer gives the desired response according to the target system (Rahman et al 2011; Kouda et al 2011a, b; Phani \& Mummadi 2005).

This research was applied by the use of sigmoid function as an activation function in order to calculate the hidden layer output and the linear function to calculate the output (Kouda et al 2011a, b; Giordano et al 2011; Han et al 2004).

In order to model electrical properties of solar cell, ANNs have been used to approximate the desired output. The flowchart of our proposed approach to find the optimal parameters using experimental data is detailed in figure 3. Approximation technique consists of three steps (Choice of neuronal structure, Learning and Validation). 
After many tests of different ANN architectures, multilayer perceptron (MLP) with two hidden layers have been considered for best results; four neurons and Logsig as transfer function for the first layer, five neurons and the Logsig as transfer function for the second layer and the Linear transfer function for the output layer.

Table 1 summarizes parameters of neural networks where the data loading is: training base, test base, validation base, number of layers and neurons, type of the transfer functions, number of iteration and estimate threshold.

\subsection{Implementation ANN in PSpice}

By using the Analog Behavioral Modeling (ABM) PSpice Library components and the results (optimal architecture, bias and weights of the network), the Solar cell model designed previously is implemented as a component in the PSpice simulator library. In order to test its effect on electrical environment, figure 4 shows the model of ABM architecture where each neuron of the ANN is replaced by one ABM which is characterized by the neuron equation. Therefore,

\section{$\downarrow \downarrow \downarrow$}

$\mathrm{Ni} / \mathrm{Au}$

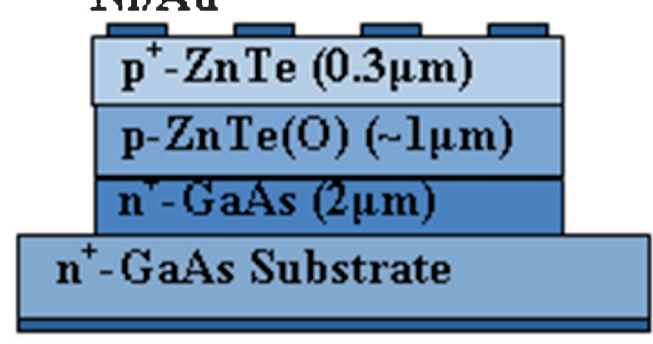

\section{Ti/Au}

Figure 1. Intermediate band solar cells structure.

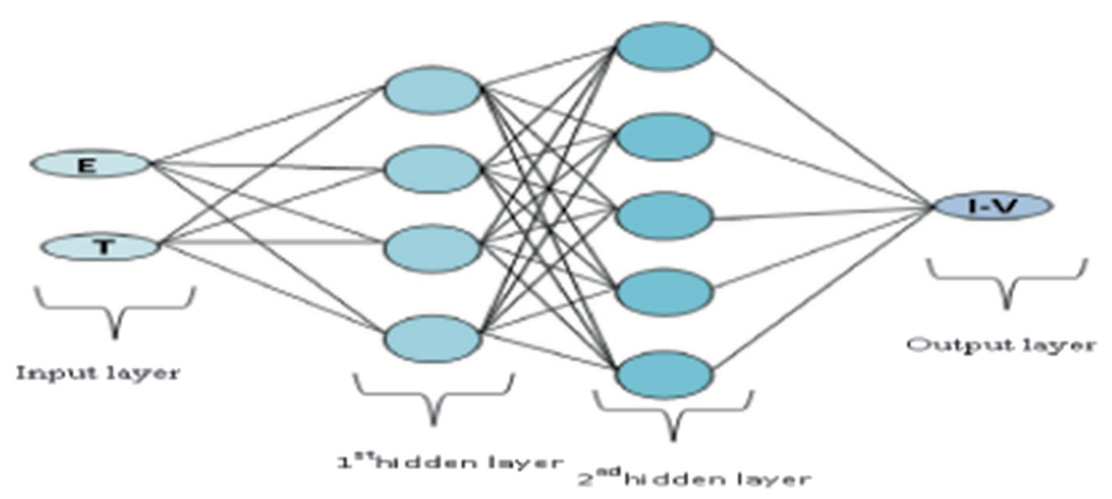

Figure 2. A typical layer neural network. 


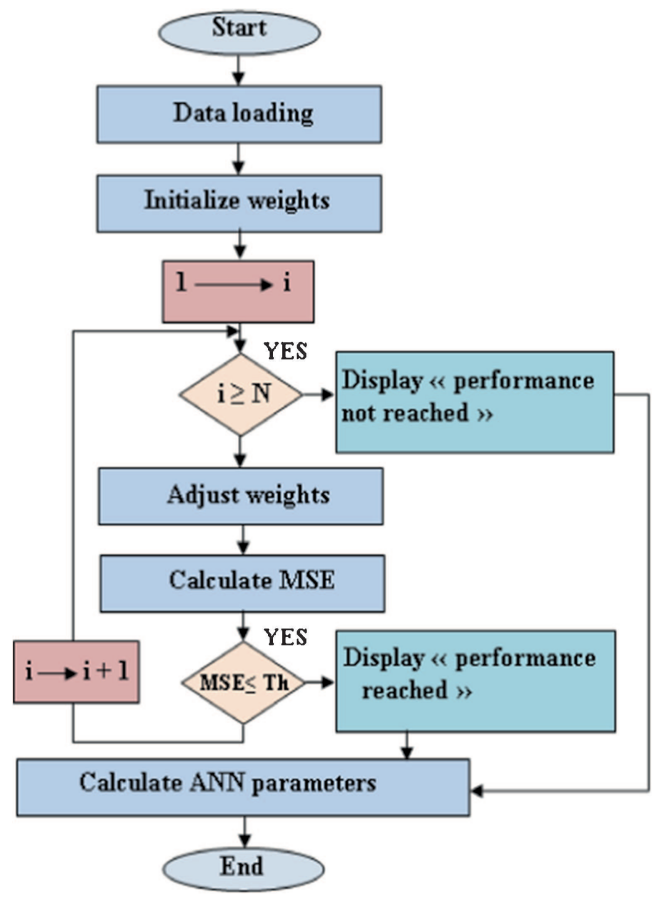

Figure 3. Training program flowchart.

Table 1. Parameters of the neural networks model.

\begin{tabular}{|c|c|c|c|c|}
\hline \multicolumn{2}{|c|}{ Database } & \multicolumn{2}{|c|}{ Number of neurons } & \multirow[t]{2}{*}{ Transfer function } \\
\hline Training base & 300 & Input layer & 02 & \\
\hline Test base & 100 & 1st hidden layer & 04 & Log sig \\
\hline Validation base & 100 & 2nd hidden layer & 05 & Log sig \\
\hline & & Output layer & 01 & Linear \\
\hline
\end{tabular}

the general form of equation in the first and second hidden layers of ABM's can be given as

$$
\text { Out i }=1 /\left(1+\exp \left(-\left(\text { Bni }+ \text { Wnij }^{*} V(I N j)+\text { Wnij }^{*} V(I N j)+\cdots+\text { Wnij }{ }^{*} V(I N j)\right)\right)\right) \text {. }
$$

For example the equation of ABM1 can be given as

$$
\text { Out } 1=1 /\left(1+\exp \left(-\left(\mathrm{B} 11+\mathrm{W} 111^{*} \mathrm{~V}(\mathrm{IN} 1)+\mathrm{W} 112^{*} \mathrm{~V}(\mathrm{IN} 2)\right)\right)\right) \text {. }
$$

The equation exponential form is due to the choice of the transfer function in the first hidden layer, B11 is the first bias of the first hidden layer in the bias matrix "Bni", where $n$ represents the number of hidden layers and $i$ is the number of ABM in the first hidden layer $(i=1 \ldots 4)$. $\mathrm{W} 111$, and W112 are respectively the first and the second weight for the first hidden layer in the weights matrix "Wnij", where $j$ varies from 1 to number of inputs ( $j=1 \ldots$ number of input). IN1 and IN2 represent input parameters which are temperature and irradiance respectively. Thus we have 10 equations with 10 bias $(4+5+1)$ and 33 weights $[(4 \times 2)+(5 \times 4)+(1 \times 5)]$. 


\section{Results and discussion}

In order to validate our proposed ANN model, we have introduced it on PSpice simulator with fixed parameters (the temperature is fixed at $300 \mathrm{~K}$ and under AM1.5 illumination E), experimental set was compared with ANN model. Figure 5 represents a comparison of the variation of current as function as output voltage using ANN model and one reported in (Weiming et al 2009) at fixed parameters. We can see that proposed model provides a good agreement between them. Figure 6 shows the variation of current versus output voltage by implementation of our proposed ANN model in PSpice simulator. Using PARAMETRIC DC SWEEP analysis, we can also observe a good agreement between experimental and results provided by PSpice simulator.

The obtained results of our proposed model are presented in table 2, where MSE is the mean square error, Th is the estimate threshold "Test MSE", Isc represents the short circuit current and $V o c$ represents the open circuit voltage. As can be seen from the table a good agreement

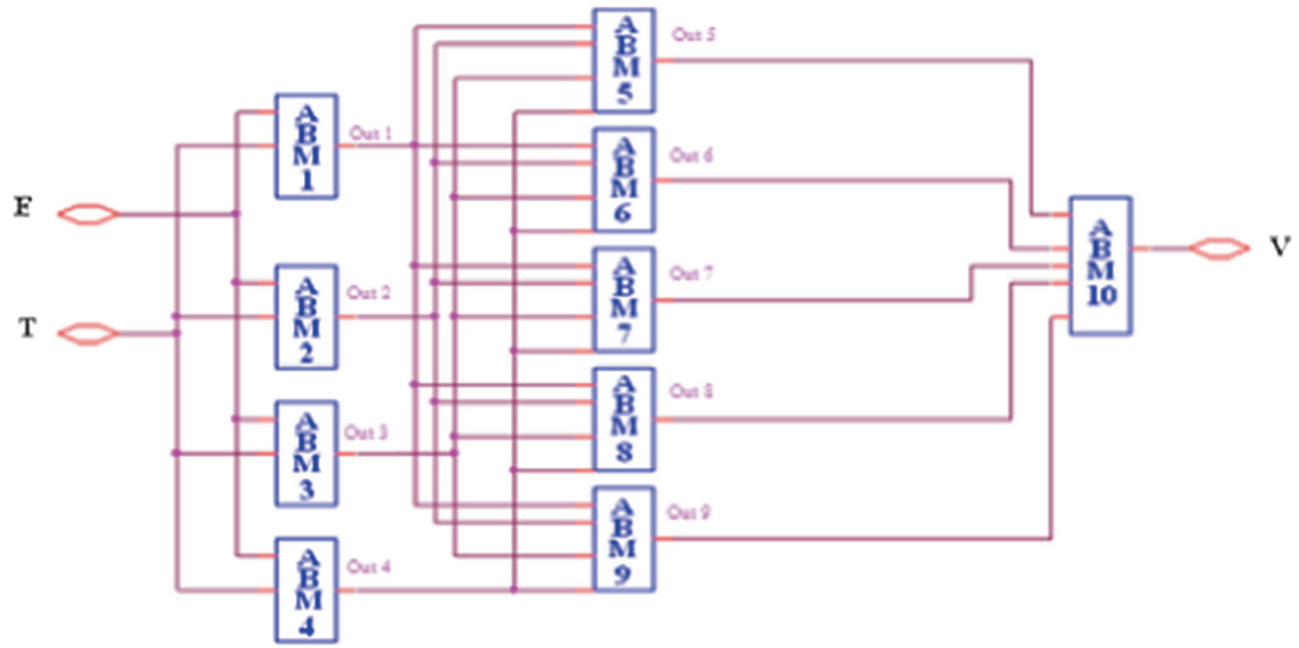

Figure 4. ABM architecture model in PSPICE.

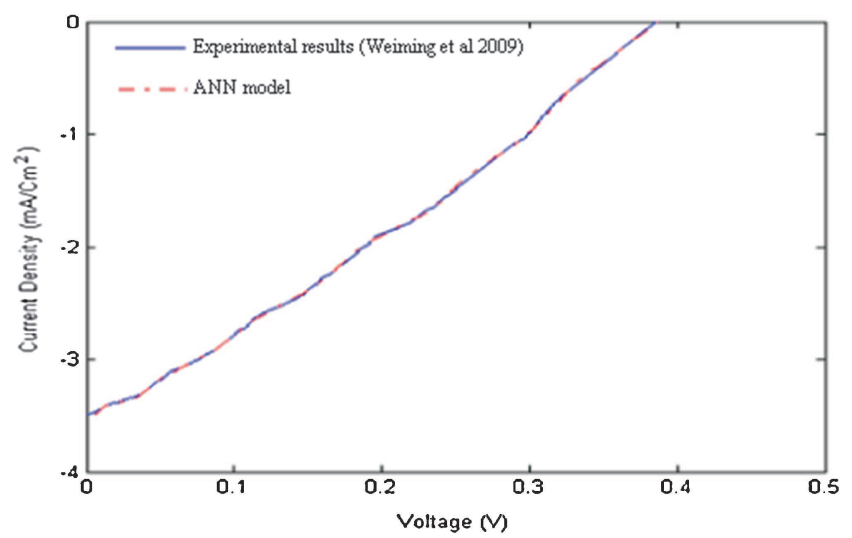

Figure 5. Variation of current density as function as output voltage with fixed parameters; $\mathrm{T}=300 \mathrm{~K}$ and $\mathrm{E}=\mathrm{AM} 1.5$. 


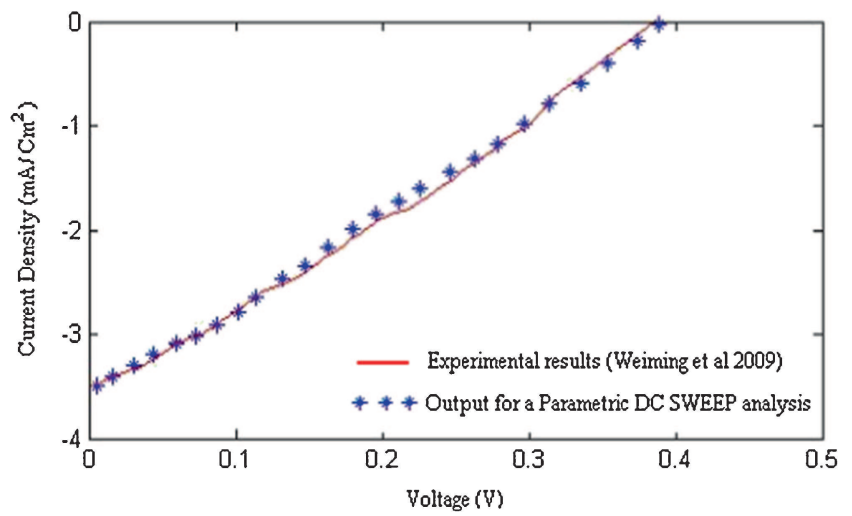

Figure 6. Variation of current density as function as output voltage with fixed parameters; $\mathrm{T}=300 \mathrm{~K}$ and $\mathrm{E}=\mathrm{AM} 1.5$ using PSpice simulator.

Table 2. Comparison with simulation results and experimental one.

\begin{tabular}{rccccccc}
\hline Input & \multicolumn{3}{c}{ Our proposed model } & \multicolumn{2}{c}{ Experimental results } & \multicolumn{2}{c}{ Mean square error } \\
\hline Illumination (E) & AM1.5 & Voc (v) & 0.39 & Voc $(\mathrm{v})$ & 0.38 & Test MSE & $3.910^{-4}$ \\
Temperature (T) & $300 \mathrm{~K}$ & Isc $\left(\mathrm{mA} / \mathrm{cm}^{2}\right)$ & 3.5 & Isc $\left(\mathrm{mA} / \mathrm{cm}^{2}\right)$ & 3.6 & Training MSE & $10^{-4}$ \\
\hline
\end{tabular}

is obtained between simulation results and experimental one. Hence, the proposed model can be used to predict other combinations of input variables (temperature and illumination) in full range. Further, the parametric simulation shows that our proposed model can be implemented as a circuit in a PSpice library to study the IBSCs-based devices for low cost and high efficiency.

\section{Conclusion}

The paper presents the modeling of I-V characteristics of IBSC based on $\mathrm{Zn}-\mathrm{Te}: \mathrm{O}$ depending on temperature and irradiance of the environment. The proposed model helps to realize the contribution of the inputs and activity of complete solar cell. The simulation results presented in this work are the evidence of the models validity and it is performed by considering an approach for modeling of IBSCS which can be done by implementation of ANN model in PSPICE. The model results have been verified and validated using experimental measurements, where a good agreement is observed between them. In addition, our study can be extended and improved by including the effect of other parameters such as serial and shunt resistances where new models must be considered. Moreover, this model may serve as a basis for a further efforts to develop $\mathrm{Zn}-\mathrm{Te}: \mathrm{O}$ and other dilute alloys or impurity materials for enhanced solar cell conversion efficiency.

\section{References}

Bremner S P and Honsberg C B 2012 Intermediate band solar cell with non-ideal band structure under AM1.5 spectrum. Photovoltaic Specialists Conference IEEE 21-24 
Giordano F, Guidobaldi A, Petrolati E, Mastroianni S, Brown T M, Reale A and Di Carlo A 2011 PSpice models for dye solar cells and modules. Numerical simulation of optoelectronic fevices (NUSOD) 10 : $43-44$

Han L, Koide N, Chiba Y and Mitate T 2004 Modeling of an equivalent circuit for dye-sensitized solar cells. Appl. Phys. Lett. 84(13): 2433

Huan-Liang Tsai, Ci-Siang Tu and Yi-Jie Su 2008 Development of generalized photovoltaic model using MATLAB/SIMULINK. Proceedings of the world congress on engineering and computer science, San Francisco

Kalogirou S A 2000 Artificial neural networks in renewable energy systems applications. Renew. Sustain. Energy Rev. 5(4): 373-401

Khelifia S, Verschraegen J, Burgelman M and Belghachia A 2008 Numerical simulation of the impurity photovoltaic effect in silicon solar cells. Renew. Energy 33(2): 293-298

Kouda Souhil, Dibi Zohir, Barra Samir, Dendouga Abdelghani and Meddour Fayçal 2011a ANN modeling of a smart MEMS-based capacitive humidity sensor. Int J. Control Autom. Syst. Springer 9(1): 197202

Kouda Souhil, Dibi Zohir, Meddour Fayçal, Barra Samir and Dendouga Abdelghani 2011b Optimization of $\mathrm{TiO} 2$ concentration effect for a chemical humidity sensing mechanism. Sensor Rev. 31(1): $18-25$

Lin A S, Wang W and Phillips J D 2009 Model for intermediate band solar cells Incorporating carrier transport and recombination and application to ZnTeO. J. Appl. Phys. 105(6): 064512-8

Lopes C L A and Lienhardt A M 2003 A simplified nonlinear power source for simulating PV panels. Power electronics specialist, IEEE 34th annual conference 04: 1729-1734

Luque A and Mart1 A 1997 Increasing the efficiency of ideal solar cells by photon induced transitions at intermediate levels. Phys. Rev. Lett. 78(26): 5014-5017

Luque A, Martí A and Cuadra L 2001 Thermodynamic consistency of sub-band gap absorbing solar cell proposals. IEEE Trans. Electron Dev. 48(9): 2118-2124

Luque A, Martí A, Stanley C, López N, Cuadra L, Zhou D and Mc-Kee A 2004 General equivalent circuit for intermediate band devices: Potentials, currents and electroluminescence. J. Appl. Phys. 96(1): 903909

Luque A, Mellor A, Ramiro I, Antolín E, Tobías I and Martí A 2013 Interband absorption of photons by extended states in intermediate band solar cells. Sol. Energy Mater. Sol. Cells 115: 138-144

Martí A and Luque A 2004 Next generation photovoltaics: High efficiency through full spectrum utilization. Institute of Physics Publishing, Bristol

Mekki H, Mellit A, Salhi H and Belhout K 2007 Modeling and simulation of photovoltaic panel based on artificial neural networks and VHDL-language. 4th International conference on computer integrated manufacturing IEEE 58-61

Moufdi H, Smail B and Hamza A 2012 Neural network for modeling solar panel. Int. J. Energy 6(1): 09-16

Nozik A J 2006 Quantum structured solar cells. Nanostructured materials for solar energy conversion 485516

Phani Kiranmai K S and Mummadi Veerachary 2005 Maximum power point tracking: A PSpice circuit simulator approach. Power electronics and drives systems, PEDS International conference 2: 1072-1077

Rahman M T, Mansur A A, Mahmud N A, Islam M and Jamal T 2011 Development of electrical behavioral model of an arbitrary solar cell to amend the PSPICE simulation performance. Power Energy Syst. IEEE $1-5$

Schokley and Queisser 1961 Detailed balance limit of efficiency of p-n junction solar cell. J. Appl. Phys. 41(3): 510-519

Stanko Tomić, Nicholas M, Harrison Timothy and Jones S 2008. Opt. Quantum Electron. Springer 40(5-6): 313-318

Tobías I, Luque A and Martí A 2011 Numerical modeling of intermediate band solar cells. Semicond. Sci. Technol. IOP publishing 26(1): 1-8

Weiming Wang, Lin Albert S and Phillips J D 2009 Intermediate-band photovoltaic solar cell based on ZnTe:O. Appl. Phys. Lett. 95(1): 011103-3 